\title{
MULHER E AIDS: PERFIL DE UMA POPULAÇÃO INFECTADA E REFLEXÕES SOBRE SUAS IMPLICAÇṌES SOCIAIS
}

\author{
Renata Ferreira Takahashi* \\ Hisako Shima** \\ Márcia de Souza***
}

\begin{abstract}
TAKAHASHI, R.F.; SHIMA, H.; SOUZA, M. Mulher e AIDS: perfil de uma população infectada e reflexões sobre
\end{abstract} suas implicações sociais. Rev. latino-am.enfermagem, Ribeirão Preto, v. 6, n. 5, p. 59-65, dezembro 1998.

O estudo teve como finalidade traçar o perfil das mulheres com AIDS notificadas ao Centro de Vigilância Epidemiológica pelo Instituto de Infectologia Emílio Ribas, no periodo de outubro a dezembro de 1995. Os dados obtidos dos prontuários médicos permitiram caracterizar o perfil em termos de idade, escolaridade, estado civil, ocupação, forma de exposição e tempo médio de sobrevida. Foi ainda possivel dimensionar o número de filhos, reais ou potenciais órfãos da AIDS, um importante problema decorrente do fenomeno. As autoras discutem as especificidades das mulheres no vivenciamento do processo infeccioso e as implicações sociais do alastramento da epidemia no grupo estudado.

UNITERMOS: AIDS, mulher, perfil, implicações sociais

\section{INTRODUÇÃO}

As estatísticas relativas à Síndrome da Imunodeficiência Adquirida (AIDS) disponíveis em nosso país denunciam claramente não só uma situação atual bastante grave, mas sobretudo um quadro futuro assustador.

$\mathrm{O}$ quadro epidemiológico da AIDS tem sofrido mudanças nos últimos anos, relacionadas tanto à forma de transmissão como ao perfil dos seus infectados. Tais mudanças dizem respeito às alterações ocorridas no interior das duas categorias de transmissão - sexual e sangüínea. Apesar, de estar sendo possível observar um decréscimo na incidência da AIDS no Estado de São Paulo, a partir de 1993 (SÃO PAULO, 1997) o Brasil ainda é um dos países com maior número de casos, pois ocupa o $3^{\circ}$ lugar no mundo (WHO, 1996).

Assim como ocorre no cenário mundial, não há como ignorar o fato de que o HIV encontra-se alastrado em nosso meio, tornando a epidemia um risco para a população em geral, como vem sendo alertado repetidamente por inúmeros pesquisadores.

Concretizam-se cada vez mais as previsões de que a infecção pelo HIV avançará mais rapidamente nos países do Terceiro Mundo onde se estima que concentrarse-ão $90 \%$ do total de casos mundiais ao redor dos anos 2000 a 2010 (FUNDAÇÃO GETÚLIO VARGAS, 1991;
MESQUITA, 1991).

No Brasil, é alarmante a tendência atual de pauperização da epidemia que pode ser constatada pelo aumento no número de casos em áreas periféricas dos centros urbanos e entre os segmentos menos privilegiados da população (GRANGEIRO, 1994; CENTRAL ÚNICA DOS TRABALHADORES, 1994), justamente entre aqueles que dispõem de menos recursos e, portanto, de menores possibilidades para o seu enfrentamento.

Outra mudança marcante que se verifica é a alteração na proporção entre homens e mulheres atingidos pelo vírus. A razão homem:mulher que era de 125:1 em 1984, passou a 4:1 ao redor de 1991-1992 e, até maio de 1997, já se somava no país, um total de 22.659 casos de AIDS na população feminina (BRASIL, 1997).

O uso de drogas injetáveis foi a principal forma de exposição até 1990 mas a partir daí, observou-se um aumento na proporção de mulheres contaminadas através de relações heterossexuais, mais da metade delas por parceiros usuários de drogas endovenosas (SANTOS \& BARBIERI, 1994).

No Estado de São Paulo, que concentra o maior número de casos de AIDS do país, SANTOS (1994) identificou, no período de janeiro de 1983 à julho de 1992, um total de 2.759 casos notificados de AIDS em mulheres acima de 10 anos de idade, sendo mais de $2 / 3$ deles na região da Grande São Paulo. No Município de São Paulo,

\footnotetext{
* Assistente do Departamento de Enfermagem em Saúde Coletiva da Escola de Enfermagem da Universidade de São Paulo

** Professora Doutora do Departamento de Enfermagem em Saúde Coletiva da Escola de Enfermagem da Universidade de São Paulo

*** Diretora da Divisão de Enfermagem do Instituto de Infectologia Emílio Ribas da Secretaria de Estado da Saúde de São Paulo
} 
a AIDS constitui-se na principal causa de morte entre mulheres de 15 a 35 anos de idade (SÃO PAULO, 1997).

A presença cada vez mais marcante da mulher no quadro da epidemia de AIDS traz a necessidade de melhor explorar a temática, pois trata-se de um segmento com especificidades distintas da população masculina e que apresenta desvantagens no que se refere à prevenção, ao controle e tratamento da infecção.

Nesse sentido, o presente estudo buscou traçar o perfil das mulheres com AIDS, tendo por base os casos notificados pelo Instituto de Infectologia Emílio Ribas (IIER) da Secretaria de Estado da Saúde de São Paulo ao Centro de Vigilância Epidemiológica de São Paulo, e refletir sobre as implicações sociais decorrentes do avanço da infecção entre a população feminina.

\section{METODOLOGIA}

Trabalhou-se com os prontuários clínicos de mulheres com AIDS, atendidas no Ambulatório do IIER e por ele notificados ao Centro de Vigilância Epidemiológica Alexandre Vranjac da Secretaria de Estado da Saúde de São Paulo, no período de outubro a dezembro de 1995.

A partir da listagem acima, fornecida pelo Serviço de Epidemiologia do IIER, foram localizados os prontuários médicos e colhidas as informações existentes e necessárias à caracterização da população em estudo, durante o mês de junho de 1996.

Vale ressaltar que tal população corresponde a uma parte da demanda total de mulheres portadoras do vírus atendidas no ambulatório do IIER, pois uma outra parcela busca o serviço já tendo sido diagnosticada e notificada por outras unidades de saúde.

Embora não tenha sido possível determinar a proporção exata, é certo que se trata de uma grande parte da demanda, pois temos constatado em outros estudos (NICHIATA, 1995; SHIMA et al., 1997) que uma proporção considerável das notificações dos casos de AIDS no Município de São Paulo tem sido feita pelo IIER e pelo Centro de Referência e Treinamento em AIDS (CRTA). Além disso, ao se comparar o número anual de notificações encaminhadas pelo IIER, com o total de casos notificados no Município de São Paulo por ano, percebe-se que praticamente metade deles se refere a notificações feitas pelo IIER $* * * *$.

Em se tratando de uma população assistida por diferentes profissionais, a falta de uniformidade no registro das informações dificultou e mesmo comprometeu a coleta de alguns dados, em particular os que se referem ao número e à idade dos filhos dessas mulheres, que possivelmente foram subestimados.

Os dados coletados foram trabalhados utilizando- se o programa D-Base III Plus e Epi-Info 5.0.

Para caracterizar a população de estudo segundo o tipo de atividade ou ocupação, utilizou-se a Classificação Brasileira de Ocupações (BRASIL, 1994a) que adota várias categorias ocupacionais considerando a analogia com os conteúdos de trabalho e as condições exigidas para o seu desempenho.

\section{RESULTADOS E DISCUSSÃO}

No curto período de 3 meses, foram detectados e notificados pelo IIER, um total de 108 casos; no entanto, analisou-se 105 casos devido à dificuldade de acesso aos prontuário no decorrer da coleta de dados.

A caracterização da população estudada segundo faixa etária, nível de escolaridade e estado civil encontrase na Tabela 1.

No grupo estudado, a idade variou de 17 a 72 anos, sendo que praticamente a metade das mulheres encontrava-se na faixa entre 26 e 35 anos, de modo que a média da idade foi de 33,9 anos e a moda situou-se nos 26 anos.

Embora a análise do grau de escolaridade tenha sido prejudicada devido à inexistência de registros adequados, foi possível perceber entre aquelas que possuíam registro, uma maior concentração de mulheres com no máximo o primeiro grau completo de escolaridade.

Tabela 1 - Distribuição das mulheres com AIDS segundo faixa etária, escolaridade e estado civil. São Paulo, Instituto de Infectologia Emílio Ribas, outubro a dezembro de 1995

\begin{tabular}{|c|c|c|c|}
\hline \multicolumn{2}{|c|}{ DADOS } & $N^{0}$ & $\%$ \\
\hline Faixa etána (anos) & $\begin{array}{l}- \text { de } 20 \\
21|--| 25 \\
26|--| 30 \\
31 \mid--35 \\
36|--| 40 \\
41|--| 45 \\
46 \mid--150 \\
51 \text { ou }+ \\
\text { sem informaçăo } \\
\text { Total }\end{array}$ & $\begin{array}{l}3 \\
14 \\
26 \\
24 \\
16 \\
7 \\
7 \\
7 \\
1 \\
105\end{array}$ & $\begin{array}{c}2,9 \\
13,3 \\
24,8 \\
22,8 \\
15,2 \\
6,7 \\
6,7 \\
6,7 \\
0,9 \\
\mathbf{1 0 0 , 0}\end{array}$ \\
\hline Escolaridade & $\begin{array}{l}\text { - analfabeta } \\
-1^{\circ} \text { grau incompleto } \\
-1^{\circ} \text { grau completo } \\
-2^{\circ} \text { grau incompleto } \\
-2^{\circ} \text { grau cormpleto } \\
\text { - sern informação } \\
\text { Total }\end{array}$ & $\begin{array}{l}1 \\
16 \\
26 \\
4 \\
6 \\
52 \\
105\end{array}$ & $\begin{array}{c}0,9 \\
15,2 \\
24,8 \\
3,8 \\
5,7 \\
49,6 \\
100,0\end{array}$ \\
\hline Estado crivil & $\begin{array}{l}\text { - casada } \\
\text { - solteira } \\
\text { - separada } \\
\text { - viưva } \\
\text { sem informação } \\
\qquad \text { Total }\end{array}$ & $\begin{array}{c}18 \\
58 \\
6 \\
20 \\
3 \\
105\end{array}$ & $\begin{array}{c}17,1 \\
55,3 \\
5,7 \\
19,0 \\
2,9 \\
\mathbf{1 0 0 , 0}\end{array}$ \\
\hline
\end{tabular}

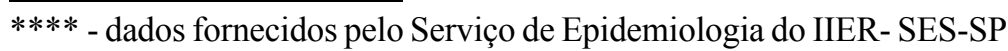


Quanto ao estado civil, constatou-se que mais da metade é solteira, sendo bastante semelhante a proporção de mulheres casadas e viúvas.

Em seu estudo com mulheres notificadas com AIDS no Estado de São Paulo, SANTOS (1994) registrou dados bastante similares no que se refere à faixa etária idade média de 31,6 anos e moda de 25 anos. O mesmo ocorreu com relação à escolaridade; no entanto, por ter considerado o estado marital e não o civil, constatou proporção menor de solteiras $(39,7 \%)$ e maior de casadas e amasiadas $(26,7 \%)$.

Cabe ressaltar, porém, a significativa diferença no percentual de viúvas: enquanto no presente estudo $19,0 \%$ das mulheres eram viúvas, no estudo de SANTOS (1994) essa proporção foi de 6,4\%. Vale especular se esse aumento se deve ao tempo decorrido do estudo da autora até o presente levantamento - mais de 3 anos.

Agrupando-se as mulheres segundo o tipo de ocupação ou atividade exercida, constatou-se a distribuição apresentada na Tabela 2. Verifica-se que $54,2 \%$ das mulheres exercem ou exerciam atividade fora do lar e que $41,0 \%$ delas dedicam-se ou dedicavam-se às prendas domésticas.

Tabela 2 - Distribuição das mulheres com AIDS segundo ocupação/atividade. São Paulo, Instituto de Infectologia Emílio Ribas, outubro a dezembro de 1995

\begin{tabular}{l|cc}
\hline \multicolumn{1}{c|}{ OCUPAÇÃO/ATIVIDADE } & N & \% \\
\hline Prendas domésticas & 43 & 41,0 \\
Atividades técricas & 19 & 18,1 \\
Comércio & 15 & 14,3 \\
Serviços e serventia & 13 & 12,3 \\
Atividades administrativas & 6 & 5,7 \\
Direção e gerência & 2 & 1,9 \\
Outras* & 2 & 1,9 \\
Sern informação & 5 & 4,8 \\
\hline \multicolumn{1}{c}{ Total } & $\mathbf{1 0 5}$ & $\mathbf{1 0 0 , 0}$ \\
\hline
\end{tabular}

* - uma estudante e uma prostituta

Quanto à forma de exposição ao HIV (Tabela $3)$, a maioria $(65,7 \%)$ referiu uma única forma, sendo marcante a importância da transmissão através de relações heterossexuais $(50,5 \%)$. Um pequeno número fez menção a formas múltiplas de exposição, invariavelmente associada ao uso de drogas injetáveis.
Tabela 3 - Distribuição da população de estudo segundo forma de exposição. Instituto de Infectologia Emílio Ribas, outubro a dezembro de 1995

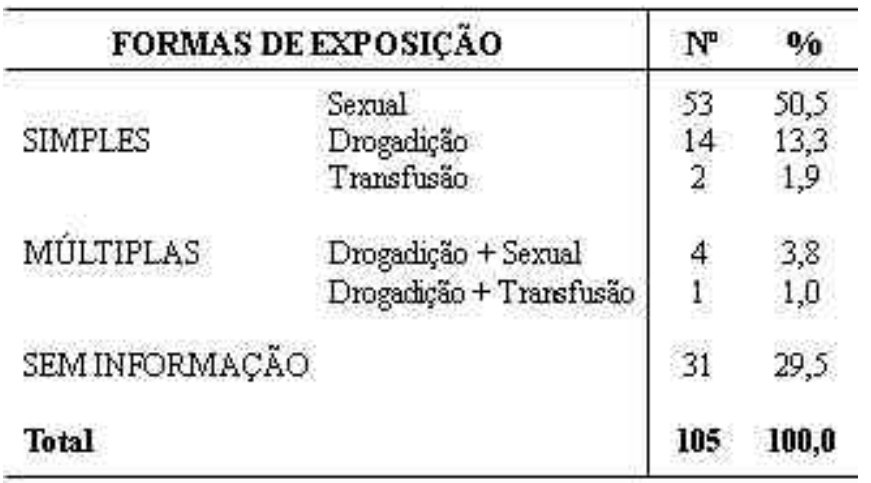

Embora no presente estudo a proporção de mulheres com forma de exposição sexual (50,5\%) tenha sido maior e o percentual de casos com transmissão através de drogas injetáveis (13,3\%) menor, quando comparados com os resultados encontrados $(43,7 \%$ e $21,5 \%$, respectivamente) por SANTOS (1994), tais achados reafirmam a mudança na forma de exposição das mulheres, conforme já vínhamos discutindo.

Tomando em conjunto as formas simples e múltiplas de exposição, verifica-se que a exposição sexual ocorreu em 57 casos $(54,3 \%)$ e a drogadição estava envolvida em 19 casos $(18,1 \%)$, reiterando, uma vez mais, a importância da contaminação através de relações heterossexuais como a forma predominante nos últimos tempos.

Além disso, das 53 mulheres com forma de exposição sexual, apenas $2(3,8 \%)$ referiram múltipla parceria, revelando que a grande maioria delas foi contaminada pelo próprio parceiro fixo, marido ou companheiro. Já naquelas 19 que se expuseram ao vírus através da drogadição ou de outras formas a ela associadas, 7 (36,8\%) indicaram múltipla parceria, sugerindo maior exposição a múltiplos contatos sexuais em um ambiente que propicia o uso drogas injetáveis.

Constatou-se ainda que $65,0 \%$ das viúvas haviam perdido o marido em decorrência da AIDS, confirmando a importância da transmissão do vírus do marido para sua esposa através de relações sexuais.

No momento da coleta de dados, 36 mulheres $(34,3 \%)$ já haviam falecido. O tempo decorrido da inscrição no serviço até o óbito é apresentado na Tabela 4. 
Tabela 4 - Distribuição de casos de óbito da população de estudo, segundo tempo decorrido após a inscrição no serviço. São Paulo, Instituto de Infectologia Emílio Ribas, outubro a dezembro de 1995

\begin{tabular}{c|cc}
\hline TEMPO & $\mathbf{N}^{\mathbf{2}}$ & $\mathbf{\%}$ \\
\hline - menos de um mês & 15 & 41,6 \\
$-1|-| 2$ meses & 8 & 22,2 \\
$-2|-| 3$ meses & 3 & 8,3 \\
$-3|-| 4$ meses & 3 & 8,3 \\
$-4--15$ meses & 2 & 5,6 \\
$-5|-| 6$ meses & 2 & 5,6 \\
-6 ou + meses & 3 & 8,4 \\
\hline Total & $\mathbf{3 6}$ & $\mathbf{1 0 0 0}$ \\
\hline
\end{tabular}

Os dados revelam que a grande maioria faleceu logo após o início do atendimento e que três clientes apresentaram uma sobrevida superior a 6 meses, sendo esta igual a 7 e 9 meses e 3 anos, respectivamente.

Por não dispor de informações sobre a data de diagnóstico da soropositividade e da assistência recebida (ou não) pelas mulheres em outras unidades de saúde no período que antecedeu o seu acesso ao IIER, os dados acima são pouco elucidativos, fazendo emergir a necessidade de melhor investigar a história e a trajetória das mulheres desde o momento do diagnóstico da soropositividade. Além disso, por se tratar de uma instituição hospitalar de referência, o IIER recebe uma grande demanda de doentes de AIDS com complicações que requer pronto atendimento ou internação, muitos em fase terminal da doença, o que pode explicar a considerável proporção de óbitos logo após o início do atendimento.

Apesar de se tratar de dados subestimados, acredita-se ser de grande importância analisar a informação sobre o número de filhos no grupo estudado, pois além de conhecer o perfil das mulheres com AIDS, buscou-se refletir sobre as implicações familiares decorrentes da sua contaminação.

Identificou-se um total de 114 filhos, indicados por 56 mulheres (Tabela 5). Dessas, $27(48,2 \%)$ eram mães solteiras ou mulheres vivendo maritalmente em uniões não formalizadas oficialmente. É possível que tais situações, com freqüência ainda não aceitas socialmente, aumentem o estigma da mulher soropositiva, dificultando ainda mais o enfrentamento da doença.

Tabela 5 - Distribuição das mulheres segundo número de filhos. São Paulo, Instituto de Infectologia Emílio Ribas, outubro a dezembro de 1995

\begin{tabular}{c|cc}
\hline N DE FLHOS & N $^{\mathbf{D}}$ DE MÃES & TOTAL DE FLHOS \\
\hline 1 & 22 & 22 \\
2 & 20 & 40 \\
3 & 8 & 24 \\
4 & 4 & 16 \\
5 & 1 & 5 \\
7 & 1 & 3 \\
\hline Total & $\mathbf{5 6}$ & $\mathbf{1 1 4}$ \\
\hline
\end{tabular}

Do total de filhos identificados, $69(60,5 \%)$ eram menores de 18 anos, sendo que deles, $36(52,2 \%)$ já eram órfãos de mãe e $6(8,7 \%)$ órfãos de pai e mãe no momento do levantamento dos prontuários médicos.

Para a mulher soropositiva, o futuro dos filhos é uma das questões que mais a angustia e aflige frente à perspectiva de não poder acompanhar o seu crescimento. Muitas manifestam em seus depoimentos a avidez com que vivenciam o tempo em comum, enquanto possível (SANTOS, 1994).

Identificou-se, ainda, um contingente de 64 pessoas, familiares de 50 das mulheres estudadas e que também estavam contaminados pelo HIV. Delas, 47 $(73,4 \%)$ eram maridos ou parceiros, $13(20,3 \%)$ eram filhos e 4 (6,3\%), irmãos. Dos 13 filhos, 5 já haviam falecido e uma das mulheres referiu ter abandonado um filho de 6 meses, portador do HIV.

Embora a maioria (37 mulheres) tenha indicado apenas um familiar infectado, 13 delas apontaram a presença de duas ou três pessoas infectadas na família.

\section{SUSCEPTIBILIDADE DA MULHER FRENTE À EPIDEMIA DE AIDS}

Diante da epidemia da AIDS, ser mulher implica em especificidades que redundam em desvantagens que geram uma situação de especial susceptibilidade em relação ao homem.

A mulher, por razões anatômicas, apresenta maior facilidade de contaminação pelo vírus. Por sua vez, os códigos normativos para prevenção, vigilância, diagnóstico e assistência foram estabelecidos em função da população masculina - em decorrência da infecção ter sido primordialmente detectada nesse grupo, de modo que são apenas adaptados para a mulher (GUIMARÃES,1994) não contemplando os sinais e sintomas específicos. Com isso, a detecção da infecção é retardada, particularmente quando não existem situações de risco, o que dificulta a suspeita por parte dos profissionais de saúde.

No caso das mulheres, BARBOSA \& VILLELA (1996) vêem uma maior possibilidade de alguns sinais e sintomas comuns à AIDS e outras doenças serem interpretados como problemas psicológicos, não sendo assim investigados em detalhe.

Tais considerações tornam-se concretas nos dados constatados por SANTOS (1994): a autora observou que o tempo médio de sobrevida após o diagnóstico foi menor (164,9 dias) nas mulheres expostas ao vírus através de relações heterossexuais do que naquelas prostitutas usuárias de droga injetáveis $(311,8$ dias) ou mulheres com parceiros bissexuais (262,6 dias).

No entanto, o diagnóstico tardio da soropositividade na mulher constitui uma questão 
problemática tanto nos países desenvolvidos como naqueles em desenvolvimento. Mesmo nos Estados Unidos onde a realidade de vida e de assistência à saúde é privilegiada em relação ao nosso país, milhares de mulheres falecem antes mesmo do diagnóstico da infecção. Também o tempo de sobrevida após o diagnóstico é bem menor do que o verificado para os homens: 15,5 semanas e 20,8 meses respectivamente (Denemberg apud BARBOSA, 1993).

Por outro lado, é possível que a mulher, ao assumir uma multiplicidade de papéis - filha, esposa, mãe, dona de casa e/ou trabalhadora -, muitas vezes relegue a um segundo plano o cuidado com o próprio corpo e com a própria saúde.

De fato, como constatou GUINSBURG (1993), muitas mulheres percebem estar infectadas somente após o parto, quando seus filhos são diagnosticados como portadores do HIV: dos 50 recém-nascidos identificados como soropositivos, apenas uma das mães estava ciente de sua condição de portadora do vírus.

Ainda, as relações de gênero que permeiam o ser e viver das mulheres na nossa realidade social interferem inclusive na sua forma de vivenciar o processo saúde-doença, implicando em susceptibilidade especial frente à epidemia da AIDS (CHECA, 1994; GUIMARÃES, 1994).

Conforme apontam BARBOSA \& VILELLA (1996), vivemos uma cultura sexual na qual as diferenças entre homens e mulheres foram convertidas em desigualdade. A dominação histórica do sexo masculino sobre o feminino, a submissão da mulher e a aceitação da sexualidade passiva da mulher faz com que a realização de sexo seguro e portanto, a prevenção contra a AIDS, se torne uma questão altamente complexa. Em primeiro lugar, porque o uso do preservativo depende da vontade do parceiro e, em segundo, pela dificuldade que a mulher tem de solicitar ao parceiro que o utilize, por suscitar desconfiança quanto à fidelidade masculina (BARBOSA, 1993).

A valorização da mulher como reprodutora, mais do que como sujeito social, reflete-se também nos serviços de assistência à saúde, que voltam suas ações basicamente para o processo gravídico, levando-se a questionar a adequação da implementação do Programa de Assistência Integral à Saúde da Mulher (PAISM).

\section{IMPLICAÇÕES SOCIAIS DO ALASTRAMENTO DA EPIDEMIA ENTRE AS MULHERES}

O presente levantamento realizado em um curto período de três meses e em um único serviço de saúde, permitiu a identificação de mais de 100 casos notificados de AIDS, somente em mulheres, o que vem reiterar a seriedade da dimensão que assume a epidemia de AIDS no nosso meio, atingindo mulheres jovens, no auge de sua vida produtiva e reprodutiva. Metade delas são mães, têm baixa escolaridade, são solteiras, trabalhadoras ou "do lar" e foram expostas ao vírus principalmente através de relações heterossexuais com maridos ou parceiros fixos.

Uma das conseqüências mais perniciosas da disseminação do HIV entre as mulheres é possivelmente a transmissão da infecção à criança durante o processo reprodutivo: $86,5 \%$ das crianças com menos de 15 anos de idade, notificadas com AIDS até 1993, haviam sido contaminadas através de transmissão perinatal do vírus (BRASIL, 1994b).

Considerando-se a principal forma de exposição, é altamente preocupante o significativo número de crianças identificadas, filhos de pais contaminados, cujo futuro certo é tornarem-se órfãos da AIDS.

No continente africano onde a epidemia da AIDS assumiu proporção catastrófica, os órfãos da AIDS vêm se revelando como uma das conseqüências sociais mais importantes da epidemia: estima-se que somente na África oriental e central, 3,1 a 5,5 milhões de crianças, o que perfaz 5 a $10 \%$ de toda a população abaixo de 15 anos de idade, tenham perdido suas mães no decorrer do decênio de 1990 (ORGANIZACION MUNDIAL DE SALUD, 1995a).

O número significativo de filhos e de familiares contaminados pelo HIV mencionado pelas mulheres permite avaliar a perversidade da infecção e o grau de desestruturação familiar, psico-emocional e mesmo financeira, que dela decorre.

Historicamente, a mulher ocupa em nossa sociedade o papel de gestora do lar, assumindo praticamente sozinha a administração e o cuidado da casa, sendo ela também quem responde pela educação e saúde dos filhos, do parceiro e muitas vezes de seus progenitores. É fácil de imaginar a desagregação familiar que virá ocorrer em conseqüência da perda desta figura central do núcleo familiar e as dificuldades econômicas que advirão se, além disso, a família não puder contar também com a figura paterna.

\section{CONSIDERAÇÕES FINAIS}

Urge reconhecer que após mais de uma década e meia do seu início, a epidemia de AIDS certamente apresenta um perfil epidemiológico bastante distinto e assim, assumir a necessidade de rever não apenas as medidas de prevenção e controle, como também a assistência aos soropositivos. Com isso, buscar-se-ão 
intervenções mais efetivas e voltadas às reais necessidades de cada grupo, segmento ou classe social, pois é cada vez mais evidente que a epidemia atinge de forma diferenciada cada um deles e que estes também a vivenciam de modo diferenciado (GRANGEIRO, 1994; NICHIATA, 1995; SHIMA et al, 1997).

No entanto, conforme denunciam PARKER \& GALVÃO (1996), um inacreditável silêncio tem envolvido a relação mulher e AIDS, acrescentando que "talvez (...) em nenhum lugar a omissão da responsabilidade em relação às mulheres e à AIDS tenha se feito tão profunda como no campo das políticas públicas e dos programas oficiais associados ao controle da AIDS no Brasil e aos serviços de saúde da mulher".

$\mathrm{O}$ enfrentamento coletivo da epidemia é imprescindível: ao lado do compromisso do poder público de enfrentar a epidemia, cabe à sociedade buscar organizar-se cada vez mais, criando alternativas para responder a novas demandas decorrentes da situação, a exemplo de outros países onde mulheres soropositivas organizaram-se para enfrentar a doença e nos quais medidas mais efetivas têm sido tomadas em relação aos órfãos da AIDS (ORGANIZACION MUNDIAL DE SALUD, 1995b).

Agradecimentos: agradecemos à FAPESP pelo auxílio parcial concedido e à Direção do Instituto de Infectologia Emílio Ribas por possibilitar a realização do presente estudo, em especial à Dra. Maria Aparecida Guerra, do Serviço de Epidemiologia.

\section{WOMAN AND AIDS: PROFILE OF AN INFECTED POPULATION AND ITS SOCIAL IMPLICATIONS}

This study aimed to identify the profile of women with AIDS from a Health Service located in São Paulo City, from October to December, 1995. The data obtained from medical charts allowed to characterize the profile in terms of age, school degree, marital status, occupation, exposed category and mean survival time. Besides, it was possible to know the number of children - sons and daughters of those women - that might be real or potential AIDS' orphans, an important social problem involving the phenomena. Authors discuss the women's particularities in the process of the disease and the social implications related to the spread of the infection in this group.

KEY WORDS: AIDS, woman, profile, social implications

\section{MUJER Y SIDA: PERFIL DE UNA POBLACIÓN INFECTADA Y IMPLICACIONES SOCIALES}

El presente estudio tuvo como finalidad conocer el perfil de las mujeres notificadas com SIDA por una institución de salud localizada en el municipio de São Paulo, de octubre a deciembre de 1995. Los datos obtenidos de los registros médicos permitieron caracterizar el perfil en términos de edad, escolaridad, estado civil, ocupación, categoría de exposición y tiempo medio de sobrevida. Además, fué posible conocer el número de hijos, reales o potenciales huérfanos de SIDA, importante problema social resultante del fenómeno. Las autoras presentan una discusión acerca de la especificidad de las mujeres en el proceso de vivir la enfermedad, así como de las implicaciones sociales de la diseminación del vírus en este grupo.

TÉRMINOS CLAVES: SIDA, mujer, perfil, implicaciones sociales

\section{REFERÊNCIAS BIBLIOGRÁFICAS}

01. BARBOSA, R.H.S. AIDS e gênero: as mulheres de uma comunidade favelada. Rio de Janeiro, 1993. 226p. Dissertação (Mestrado) - Escola Nacional de Saúde Pública, Fundação Osvaldo Cruz.

02. ; VILLELA, W.V. A trajetória feminina da AIDS. In: PARKER, R.; GALVÃO, J. Quebrando o silêncio: mulheres e AIDS no Brasil. Rio de Janeiro: Relume-Dumará, ABIA, IMS/UERJ, 1996. p.17-32.

03. SÃO PAULO. Secretaria de Estado da Saúde de São Paulo. Programa DST/AIDS. Bol. Epidemiol. AIDS. São Paulo, n.2, agosto 1997.
04. BRASIL. Ministério do Trabalho. Secretaria de Políticas de Emprego e Salários. Classificação brasileira de ocupações: índice ampliado. Brasília, 1994a.

05. BRASIL. Ministério da Saúde. Programa Nacional de Doenças Sexualmente Transmissíveis/AIDS. Bol.Epidemiol. AIDS, Brasília, v.7, n.8, 1994 b.

06. BRASIL. Ministério da Saúde. Programa Nacional de Doenças Sexualmente Transmissíveis/AIDS. Bol.Epidemiol. AIDS. Brasília, n.6, mar. 1997.

07. CENTRAL ÚNICA DOS TRABALHADORES. (BRASIL). Comissão Nacional de Prevenção à AIDS. A AIDS e os trabalhadores. São Paulo, 1994. /mimeo/ 
08. CHECA, S. Salud y genero: aproximaciones a la problemática de salud de la mujer. In: KORNBLIT, A.L. Ciencias sociales $\mathbf{y}$ medicina: la salud en Latino Americana. Buenos Aires: Instituto de Investigaciones de la Facultad de Ciencias Sociales de la Universidad de Buenos Aires, 1994. p.71-7.

09. FUNDAÇÃO GETÚLIO VARGAS. Investindo na população. In: RELATÓRIO sobre o desenvolvimento mundial- 1991: o desafio do desenvolvimento. Rio de Janeiro, 1991. cap.3, p.53-78.

10. GRANGEIRO, A. O perfil socio-econômico dos casos de AIDS na cidade de São Paulo. In: PARKER,R. et el. A AIDS no Brasil. Rio de Janeiro: Relume-Dumará, 1994. cap.7, p.91-128.

11. GUIMARÃES, C.D. ulheres, homens e AIDS: o visível e o invisível. In: PARKER,R. et al. A AIDS no Brasil. Rio de Janeiro: Relume-Dumará, 1994. cap.7, p.218-230.

12. GUINSBURG, R. et al. Sorologia positiva para sífilis no período neonatal: prevalência em maternidade de nível secundário. Associação com fatores de risco maternos e com sorologia positiva para HIV1. Rev.Assoc.Méd.Bras., v.39, n.2, p. 100-4, 1993.

13. MESQUITA, F.C. AIDS e drogas injetáveis. In: DANIEL, H. et al. Saúde \& loucura. São Paulo: Hucitec, 1991. p. 47-53.
14. NICHIATA, L.Y.I. AIDS: os (des)caminhos da informação. São Paulo, 1995. 109 p. Dissertação (Mestrado) - Escola de Enfermagem da Universidade de São Paulo.

15. ORGANIZACION MUNDIAL DE SALUD. Mujeres, sexo y SIDA. In: ORGANIZACION MUNDIAL DE SALUD SIDA: imagenes de la epidemia. Organización Mundial de Salud, Ginebra, 1995 a. cap.15, p.56-102.

16. ORGANIZACION MUNDIAL DE SALUD. Las repercusiones del SIDA. In: ORGANIZACION MUNDIAL DE SALUD SIDA: imagenes de la epidemia. Genebra: Organización Mundial de Salud, 1995 b. cap.12, p.36-8.

17. WORD HEALTH ORGANIZATION AIDS. Weekly Epidemiological Record, Geneva, v.71, p. 205-212, july 1996.

18. PARKER, R.; GALVÃO, J. Quebrando o silêncio: mulheres e AIDS no Brasil. Rio de Janeiro: Relume-Dumará, ABIA, IMS/UERJ, 1996.

19. SANTOS, N.J.S. As mulheres e a AIDS. São Paulo, 1994. 243 p. Dissertação (Mestrado) - Faculdade de Saúde Pública da Universidade de São Paulo.

20. ; BARBIERI, D.D. A AIDS e as mulheres. REAIDS, v. 2, n.1, p.1-5, 1994.

21. SHIMA, H.; AFUSO, D.S.; NICHIATA, L.Y.I. AIDS: a epidemia entre a população nipobrasileira residente no Município de São Paulo, 1997 /mimeo/. 